Vietnam Journal of Mechanics, VAST, Vol.32, No. 3 (2010), pp. $135-144$

\title{
A NEW ALGORITHM FOR KINEMATIC PROGRAM CONTROL OF OPTICAL PARTS BY GRINDING
}

\author{
Nguyen Van Khang ${ }^{1}$, Nguyen Trong Hung ${ }^{2}$ \\ ${ }^{1}$ Hanoi University of Technology \\ ${ }^{2}$ Hung Yen University of Technology and Education
}

\begin{abstract}
Based on the definition of the local coating coefficient, the average local coating coefficient and the speed coefficient in grinding of optical parts, and on theory of multibody kinematics, an algorithm for kinematic program control during the grinding process of optical parts has been developed at Hanoi University of Technology. Using this algorithm, the results of the improvement in the precision of processed surfaces of optical parts by grinding under kinematic program control have been obtained and will be presented in the present paper.
\end{abstract}

\section{INTRODUCTION}

With the development of high precision mechanic and optical sectors, optical tools and devices have been playing an important role in many industrial fields. Key components in optical tools and devices are parts made by optical glasses, hereunder called as optical parts. Among processing methods, grinding is one of the most effective methods to achieve high precision, even though the facilities are not at the same level of precision.

The problem to improve processed surface's precision of optical parts by grinding is widely interested. It's related to a lot of technological factors. The study on the influence of kinematics on precision of the processed part's surface is one of effective methods by improving processed surface's precision of optical parts.

Study on kinematic program of processed optical parts is still limited [1, 2]. By applying kinematics of multibody systems $[3,4,5,6]$, the present paper's authors established a kinematic program for processing optical parts on grinding equipment to improve processed surface's precision of optical parts.

Experiments were carried out on optical part grinding equipment with four - bar mechanism $([1,2,7])$. Consider the grinding equipment as shown in Fig. 1, in which

- $\omega_{1}$ is the angular speed of level 1 ,

- $\omega_{3}$ is the shaking speed of bar 3 ,

- $\omega_{4}$ is the angular speed of disk 4 ,

- $\omega_{5}$ is the angular speed of polishing instrument 5 , 


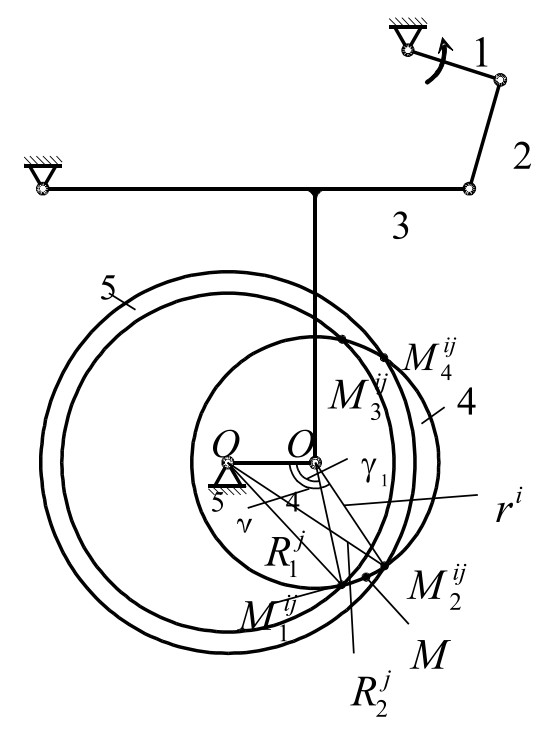

Fig. 1. The four - bar mechanism of optical part grinding equipment

- Disk 4 is for fixing the processing part.

Due to the friction between the surface of the grinding instrument and that of the processing part, the support disk does not only move with bar 3 , but also rotates relatively around center $0_{4}$ with the angular speed $\omega_{4}$.

In the case of grinding, the ratio between $\omega_{4}$ and $\omega_{5}$ or $\omega_{1}$ and $\omega_{5}$ is selected based on technology conditions: $\omega_{4} / \omega_{5}=k_{2}, \omega_{1} / \omega_{5}=k_{1}$.

\section{CONTROL OF PROCESSING KINEMATIC PROGRAM WHILE GRINDING}

The relative speed is one of the factors affecting the abrasion intensity of optical parts. In order to express relationships of abrasion intensity and the relative speed, a non dimension factor is introduced, and it is called the speed coefficient.

The factor

$$
\chi_{i j}(t)=\frac{{ }^{5} v_{i j}^{r}(t)}{V_{R \max }}
$$

is called as the speed coefficient $[1,7]$, where $V_{R \max }=\omega_{5} D_{5} / 2$ is the speed of a point on external hoop of the grinding instrument 5 , and ${ }^{5} v_{i j}^{r}(t)$ is the average relative speed of points $\mathrm{M}$ on support disk 4 in $\operatorname{arc} M_{1}^{i j} M_{2}^{i j}$ against grinding instrument $5, D_{5}$ is the diameter of the grinding instrument.

From Fig. 1, the relationship of relative velocity can be expressed by the following formula

$$
{ }^{5} v_{i j}^{r}(t)=\frac{1}{\gamma_{1}-\gamma_{2}} \int_{\gamma_{1}}^{\gamma_{2}} 5 \nu_{M}^{r} d \gamma
$$


where $\gamma_{1}(t) \leq \gamma(t) \leq \gamma_{2}(t),{ }^{5} \nu_{M}^{r}(t)$ is the speed of any point $\mathrm{M}$ in the arc $M_{1}^{i j} M_{2}^{i j}$ on support disk 4 that is defined by the following formula $[3,4,5,6]$

$$
\left[\begin{array}{c}
\dot{\xi}_{M}^{(5)} \\
\dot{\eta}_{M}^{(5)}
\end{array}\right]=\mathbf{A}_{5}^{T}\left\{\left[\begin{array}{c}
\dot{x}_{O 4} \\
\dot{y}_{O 4}
\end{array}\right]+\dot{\varphi}_{4} \mathbf{I}^{*} \mathbf{A}_{4}\left[\begin{array}{c}
\xi_{M}^{(4)} \\
\eta_{M}^{(4)}
\end{array}\right]\right\}+\dot{\varphi}_{5} \mathbf{I}^{* T} \mathbf{A}_{5}^{T}\left\{\left[\begin{array}{c}
x_{O 4} \\
y_{O 4}
\end{array}\right]-\left[\begin{array}{c}
x_{O 5} \\
y_{O 5}
\end{array}\right]+\mathbf{A}_{4}\left[\begin{array}{c}
\xi_{M}^{(4)} \\
\eta_{M}^{(4)}
\end{array}\right]\right\}
$$

In this equation the cosine directive matrices $\mathbf{A}_{i}$ and the matrix $\mathbf{I}^{*}$ have the following forms

$$
\mathbf{A}_{4}=\left[\begin{array}{cc}
\cos \varphi_{4} & -\sin \varphi_{4} \\
\sin \varphi_{4} & \cos \varphi_{4}
\end{array}\right], \quad \mathbf{A}_{5}=\left[\begin{array}{cc}
\cos \varphi_{5} & -\sin \varphi_{5} \\
\sin \varphi_{5} & \cos \varphi_{5}
\end{array}\right], \quad \mathbf{I}^{*}=\left[\begin{array}{cc}
0 & -1 \\
1 & 0
\end{array}\right]
$$

Now the concept of average speed coefficient in a cycle of the level of drive element [1] is introduced

$$
\bar{\chi}_{i j}=\frac{1}{T} \int_{0}^{T} \chi_{i j}(t) d t=\frac{1}{T V_{R \max }} \int_{0}^{T} 5 v_{i j}^{r}(t) d t
$$

Consider relative average speed coefficient of the i-th hoop of the support disk 4 against grinding instrument 5

$$
\bar{\chi}_{i}=\frac{1}{m^{*}} \sum_{k=1}^{m^{*}} \bar{\chi}_{i k}
$$

where $m^{*}$ is the quantity of hoops on grinding instrument 5 , while $\bar{\chi}_{i j} \neq 0$ and $j=1, \ldots, m$. The speed coefficient $\bar{\chi}_{i}$ and coating coefficient $\bar{C}_{i}$ exhibit the kinematic influence of grinding process of the instrument 5 on abrasion intensity of part's surface on hoops with any radius $r^{i}$ of the support disk 4 . Their influences are simultaneous, co-operating and may compensate each other.

In that case the condition for the grinding instrument 5 to smoothly process the part's surface on the support disk 4 is [1]

$$
\bar{C}_{i} \bar{\chi}_{i}=\text { const }, \quad(i=1, \ldots, n)
$$

Condition (5) is an important technology requirement. To meet condition (5), after setting kinematic program to achieve reasonable relative speed function, coating coefficient $\bar{C}_{i}$ should be adjusted. Note that in practice, coating coefficient $\bar{C}_{i}$ may be adjusted by the variation of a parameter called the coefficient of filling in instrument surface $\eta_{R j}[1]$.

\section{ALGORITHM FOR KINEMATIC PROGRAM CONTROL DURING GRINDING PROCESS OF OPTICAL PARTS}

In optical part grinding operation, grinding disk 5 rotates around center $O_{5}$, while the support disk 4 performs both shaking and rotating motions around center $O_{4}$. Based on relative positions between the grinding disk 5 and the support disk 4 as shown in Fig.1, the value of partial contact coefficient $C_{i j}$ can be defined (in accordance with formula 5) as follows $[7,8]$ :

- The support disk with radius $\mathrm{r}^{i}$ intersects the other two with radius $R_{1}^{j}$ and $R_{2}^{j}$ respectively (Fig. 2): 

$\left|\begin{array}{l}r^{i}-R_{1}^{j} \\ r^{i}-R_{2}^{j}\end{array}\right|<e<r^{i}+R_{1}^{j}$ : The disk with radius $r_{i}$ intersects the disk with radius $R_{1}^{j} ;$
$C_{i j}=\frac{1}{\pi}\left(\arccos B_{i j}-\arccos A_{i j}\right)$.

- The support disk with radius $r_{i}$ intersects the disk with radius $R_{2}^{j}$ and touches the support disk with radius $R_{1}^{j}$ (Fig. 3):

$$
\begin{aligned}
& \left|r^{i}-R_{2}^{j}\right|<e<r^{i}+R_{2}^{j} ; \quad\left|r^{i}-R_{1}^{j}\right|<e=r^{i}+R_{1}^{j} \\
& C_{i j}=\frac{1}{\pi}\left(\arccos B_{i j}-\arccos A_{i j}\right) .
\end{aligned}
$$

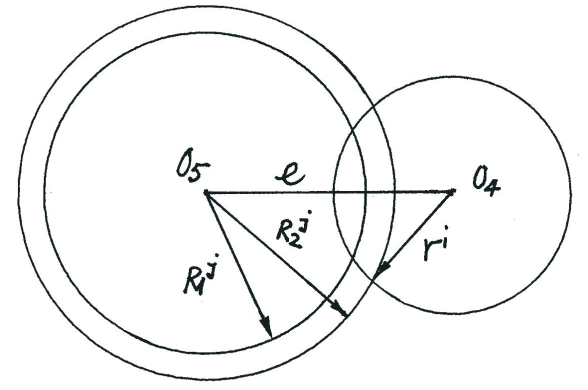

Fig. 2

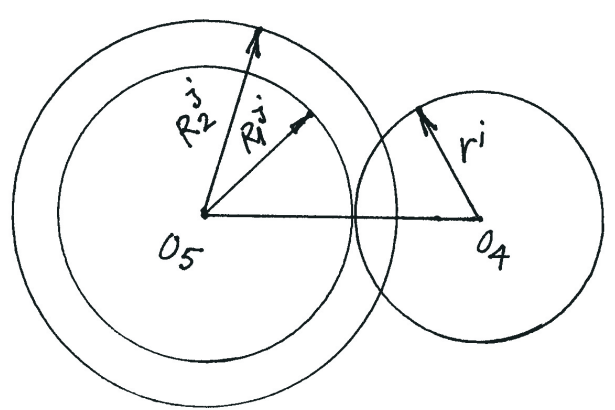

Fig. 3

- The support disk with radius $r_{i}$ intersects the disk with radius $R_{2}^{j}$ and lies entirely outside of the support disk with radius $R_{1}^{j}$ (Fig. 4):

$\left|R_{2}^{j}-r^{i}\right|<e<r^{i}+R_{2}^{j}$ : the disk with radius $r^{i}$ intersects the disk with radius $R_{2}^{j}$; $e>r^{i}+R_{1}^{j}$ : the disk with radius $r^{i}$ is not touching the disk with radius $R_{1}^{j}$; $C_{i j}=\frac{1}{\pi} \arccos B_{i j}$.

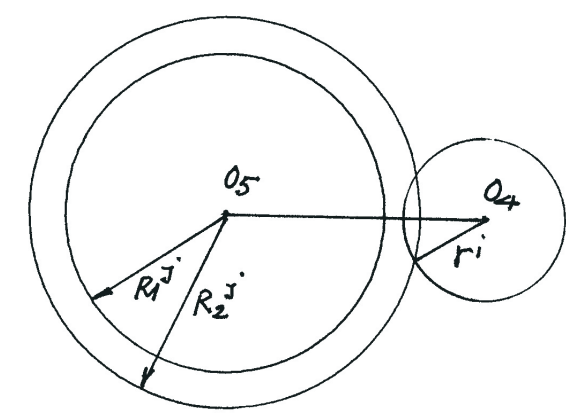

Fig. 4

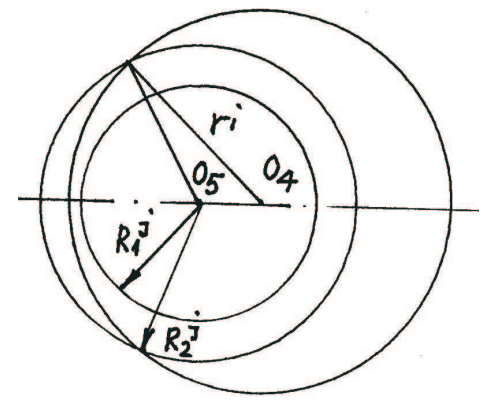

Fig. 5

- The support disk with radius $r^{i}$ intersects the disk with radius $R_{2}^{j}$ and encloses the entire disk with radius $R_{1}^{j}$ (Fig. 5): 
$\left|r^{i}-R_{2}^{j}\right|<e<r^{i}+R_{2}^{j}$ :the disk with radius $r^{i}$ intersects the disk with radius $R_{2}^{j}$;

$\left|r^{i}-R_{1}^{j}\right|>e$ : the disk with radius $r^{i}$ encloses the disk with radius $R_{1}^{j} ;\left(r^{i}-R_{1}^{j}>e\right)$;

$C_{i j}=\frac{1}{\pi} \arccos B_{i j}$.

- The support disk with radius $r^{i}$ intersects the disk with radius $R_{2}^{j}$ and simultaneously touches and encloses the disk with radius $R_{1}^{j}$ (Fig. 6):

$\left|r^{i}-R_{2}^{j}\right|<e<r^{i}+R_{2}^{j}$ : the disk with radius $r^{i}$ intersects the disk with radius $R_{2}^{j} ;$

$r^{i}-R_{1}^{j}=e$ : the disk with radius with $r^{i}$ exteriorly touches the disk with radius $R_{1}^{j}$

$C_{i j}=\frac{1}{\pi} \arccos B_{i j}$

- The support disk with radius $r^{i}$ intersects the disk with radius $R_{1}^{j}$ and lies entirely outside of the disk with radius $R_{2}^{j}$ (Fig. 7):

$\left|r^{i}-R_{1}^{j}\right|<e<r^{i}+R_{1}^{j}$ : the disk with radius $r^{i}$ intersects the disk with radius $R_{1}^{j}$;

$R_{2}^{j}-r^{i}>e$ : the disk with radius $R_{2}^{j}$ encloses the disk with radius $r^{i}$;

$C_{i j}=\frac{1}{\pi}\left(\pi-\arccos A_{i j}\right)$.

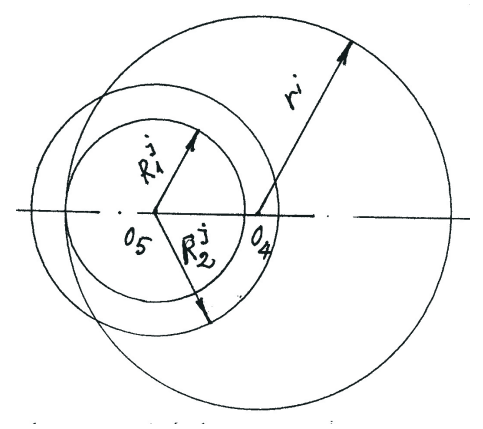

Fig. 6

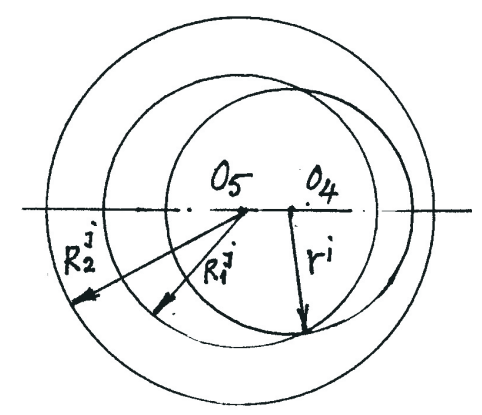

Fig. 7

- The support disk with radius $r^{i}$ intersects the disk with radius $R_{1}^{j}$ and lies entirely outside of the disk with radius $R_{2}^{j}$ (Fig. 8):

$R_{2}^{j}-r^{i}>e$ : the disk with radius $R_{2}^{j}$ encloses the disk with radius $r^{i}$;

$r^{i}+R_{1}^{j}<e$ : the disk with radius $R_{1}^{j}$ and the disk with radius $r^{i}$ are away from each other;

$C_{i j}=1 ;\left(r^{i} \leq \frac{R_{2}^{j}-R_{1}^{j}}{2}=\frac{\Delta R^{j}}{2}\right)$.

- The support disk with radius $r^{i}$ lies entirely inside the disk with radius $R_{2}^{j}$ and outside the disk with radius $R_{1}^{j}$ (Fig. 9):

$R_{2}^{j}-r^{i}>e$ : the disk with radius $R_{2}^{j}$ encloses the disk with radius $r^{i}$;

$r^{i}-R_{1}^{j}>e$ : the disk with radius $r^{i}$ encloses the disk with radius $R_{1}^{j}$;

$C_{i j}=1$. 


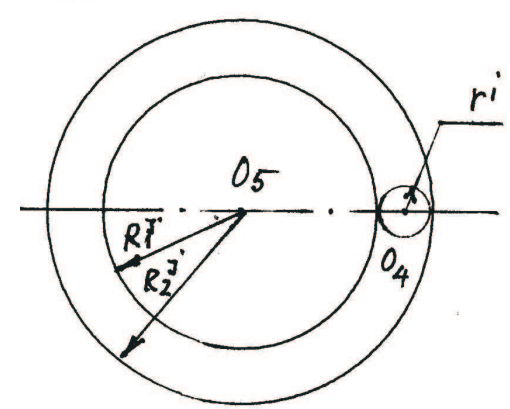

Fig. 8

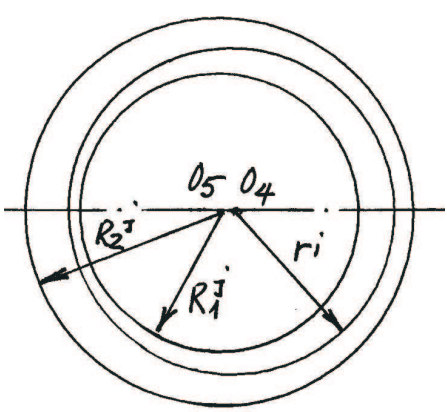

Fig. 9

- The support disk with radius $r^{i}$ lies entirely inside the disk with radius $R_{2}^{j}$ and the disk with radius $R_{1}^{j}$ (Fig. 10):

$R_{2}^{j}-r^{i}>e$ : the disk with radius $R_{2}^{j}$ encloses the disk with radius $r^{i}$;

$R_{1}^{j}-r^{i}>e$ : the disk with radius $R_{1}^{j}$ encloses the disk with radius $r^{i}$;

$C_{i j}=0, \quad{ }^{5} v_{i j}^{r}=0$.

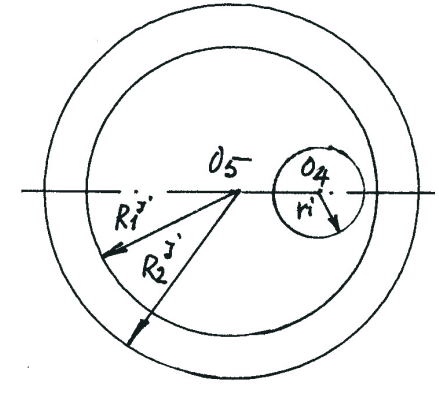

Fig. 10

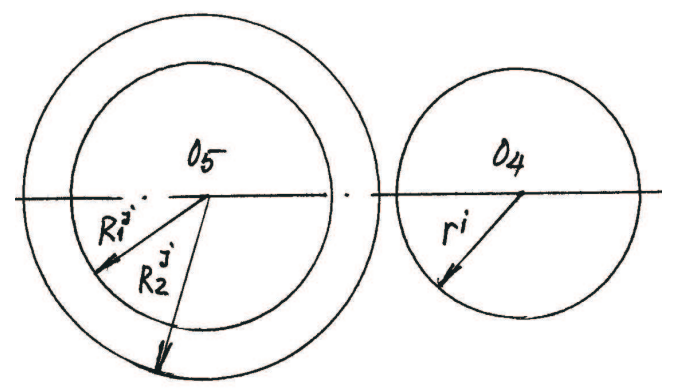

Fig. 11

- The support disk with radius $r^{i}$ lies entirely outside the disk with radius $R_{2}^{j}$ and the disk with radius $R_{1}^{j}$ (Fig. 11): other;

$r^{i}+R_{1}^{j}<e:$ the disk with radius $r^{i}$ and the disk with radius $R_{1}^{j}$ are away from each

$r^{i}+R_{2}^{j} \leq e$ : the disk with radius with $r^{i}$ and the disk with radius $R_{2}^{j}$ are away from each other;

$C_{i j}=0$.

- The support disk with radius $r^{i}$ lies entirely inside the disk with radius $R_{2}^{j}$ and outside the disk with radius $R_{1}^{j}$ (Fig. 12): other;

$r^{i}-R_{2}^{j}>e$ : the disk with radius $r^{i}$ and the disk with radius $R_{2}^{j}$ are away from each

$r^{i}-R_{1}^{j} \geq e:$ the disk with radius $r^{i}$ encloses the disk with radius $R_{1}^{j}$; 
$C_{i j}=0, \quad{ }^{5} v_{i j}^{r}=0$.

- The support disk with radius $r^{i}$ lies entirely inside the disk with radius $R_{2}^{j}$ and exteriorly touches the disk with radius $R_{1}^{j}$ (Fig. 13):

$e=0 ; r^{i}=R_{1}^{j}$ : the disk with radius $r^{i}$ exteriorly touches the disk with radius $R_{1}^{j}$;

$r^{i}<R_{2}^{j}$ : the disk with radius $r^{i}$ lies entirely within the disk with radius $R_{2}^{j}$;

$C_{i j}=0 ; \quad{ }^{5} v_{i j}^{r}=0$.

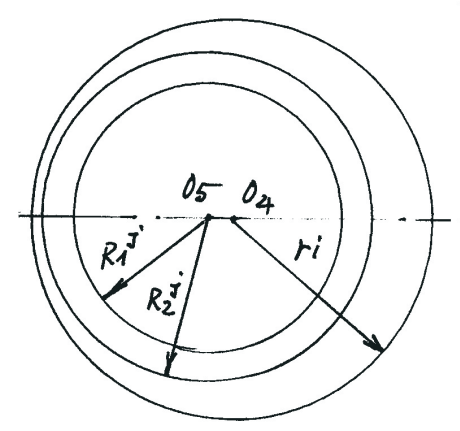

Fig. 12

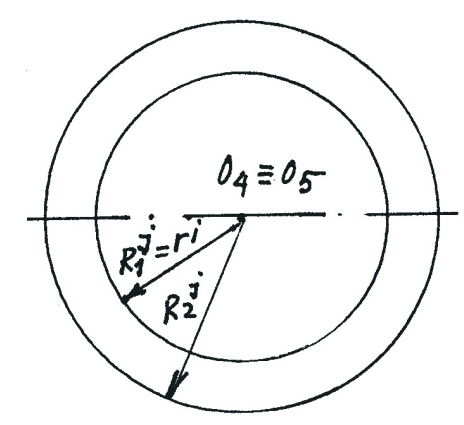

Fig. 13

- The support disk with radius $r^{i}$ exteriorly touches the disk with radius $R_{2}^{j}$ and lies completely outside the disk with radius $R_{1}^{j}$ (Fig. 14):

$e=0 ; r^{i}=R_{2}^{j}$ : the disk with radius $r^{i}$ exteriorly touches the disk with radius $R_{2}^{j} ;$ $r^{i}>R_{1}^{j}$ : the disk with radius $r^{i}$ encloses the disk with radius $R_{1}^{j}$;

$C_{i j}=0, \quad{ }^{5} v_{i j}^{r}=0$.

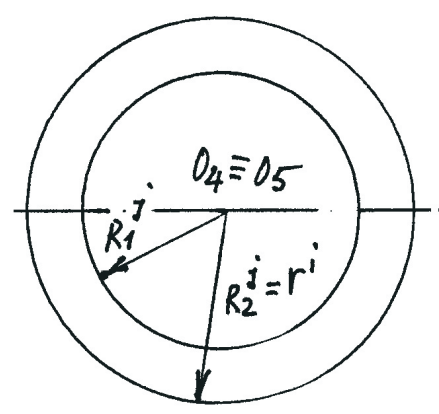

Fig. 14

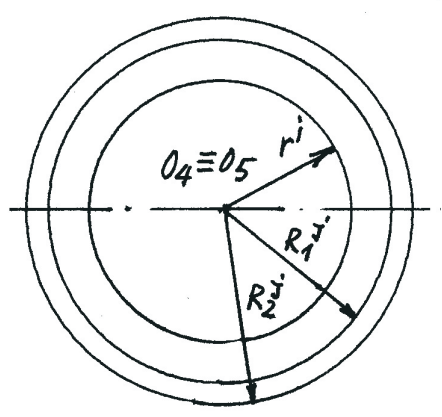

Fig. 15

- The support disk with radius $r^{i}$ lies entirely inside the disk with radius $R_{1}^{j}$ and the disk with radius $R_{2}^{j}$ (Fig. 15):

$e=0 ; r^{i}<R_{1}^{j}$ : the disk with radius $r^{i}$ lies inside the disk with radius $R_{1}^{j}$;

$r^{i}<R_{2}^{j}$ : the disk with radius $r^{i}$ lies inside the disk with radius $R_{2}^{j}$; 
$C_{i j}=0, \quad{ }^{5} v_{i j}^{r}=0$.

- The support disk with radius $r^{i}$ encloses the entire the disk with $R_{1}^{j}$ and the disk with $R_{2}^{j}$ (Fig. 16):

$e=0 ; r^{i}>R_{1}^{j}$ : the disk with radius $r^{i}$ encloses the disk with radius $R_{1}^{j}$;

$r^{i}>R_{2}^{j}$ : the disk with radius $r^{i}$ encloses the disk with radius $R_{2}^{j}$;

$C_{i j}=0 ;{ }^{5} v_{i j}^{r}=0$.

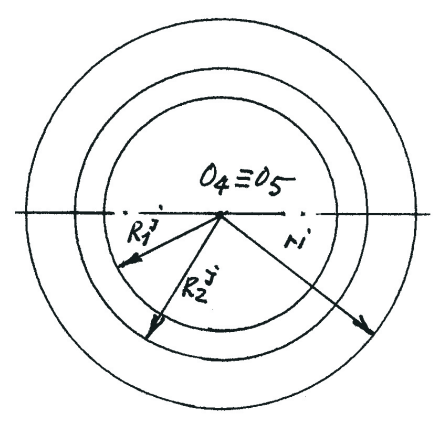

Fig. 16

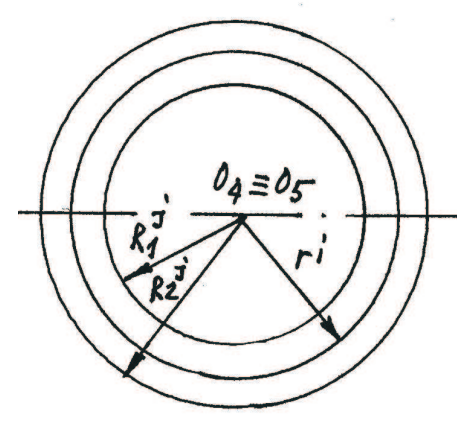

Fig. 17

- The support disk with radius $r^{i}$ lies between the disk with $R_{1}^{j}$ and the disk with $R_{2}^{j}$ (Fig. 17):

$e=0 ; \quad R_{1}^{j}<r^{i}<R_{2}^{j}$ : the disk with radius $r^{i}$ encloses the disk with radius $R_{1}^{j}$ and lies completely inside the disk with radius $R_{2}^{j} ; C_{i j}=1$.

\section{SOME RESULTS OF STUDY ON KINEMATIC PROGRAM CONTROL OF GRINDING OPTICAL PARTS}

Based on the concepts of the above mentioned coating coefficient and speed coefficient, a kinematic control program for processing parts on grinding machine type (Fig. 1) was developed. With every set of kinematic parameters of this polishing machine, a kinematic control program is formulated for respective processing parts.Hereunder were some received results of kinematic control program for processed optical parts on grinding machine (Figures 18-20).

From the investigated kinematic program of grinding optical parts surfaces following facts were recorded [7]:

- With short length of level, processing intensity at central area makes few convex spots of grinding parts.

- With medium length of level, processing intensity is relatively regular at both central area and edge area of grinding parts.

- With long length of level, processing intensity is strong at central area, so it makes sunken spots of grinding parts.

- When rough grinding, high speed of the level and high gear ratio are chosen to achieve high production capacity. 
- When pre-final grinding, medium speed of the level and medium gear ratio are chosen to achieve production capacity and precision precision.

- When final grinding, low speed of the level and small gear ratio to achieve high

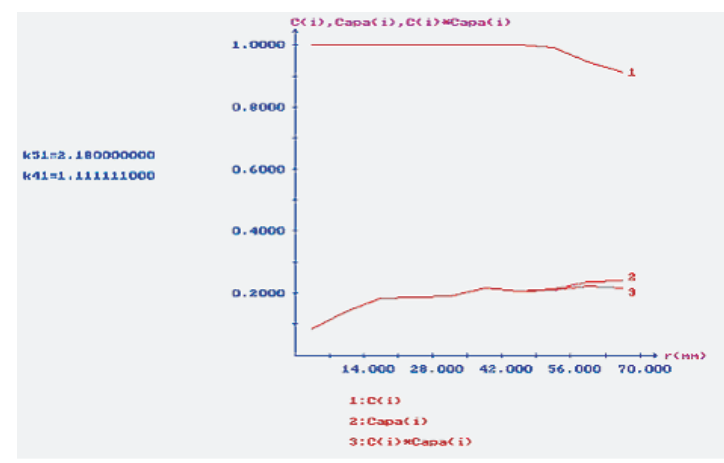

Fig. 18. Kinematic program with $l_{1}=40 \mathrm{~mm}$, relatively regular processing intensity from central to edge

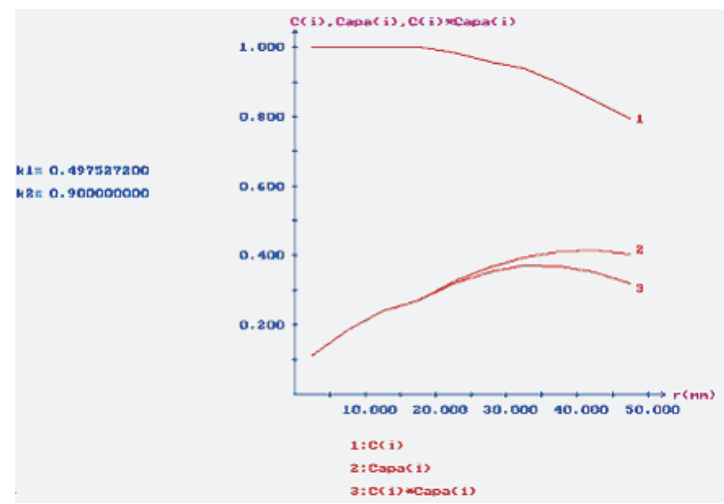

Fig. 19. Kinematic program with $l_{1}=40 \mathrm{~mm} ; k_{1}=0,4975272 ; \xi_{3}=150 ; \eta_{3}=310$

The curb expresses smooth kinematic program, reducing local vein errors

Based on established kinematic program, grinding parts by directly processing upon needed local spots can be carried out to improve precision of optical parts [7] and [8].

\section{CONCLUSION}

From the results of study on adjustment of kinematic program of grinding optical parts, the following conclusions can be made. The change of accumulative value of coating coefficient and speed coefficient depends on:

- The shaking amplitude of support disk against polishing instrument

- The variation of transformation ratio between level and main axes.

It has been shown that the control of kinematic program together with the variation of the coefficient of filling in instrument surface can improve precision of grinded surface of optical parts. 


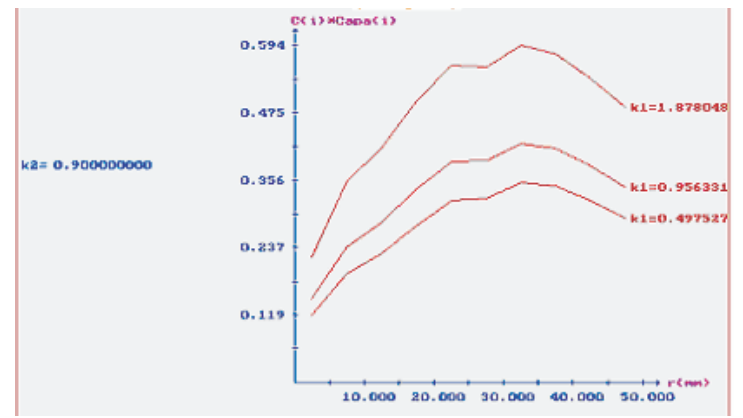

Fig. 20. Kinematic program with $l_{1}=40 \mathrm{~mm}$, and variation of parameter $k_{1}$ : Coarse grinding $k_{1}=1.87804878$ (Upper curb), Semi-fine grinding $k_{1}=0.95633187$ (Central curb),

Fine grinding $k_{1}=0,4975272$ (Lower curb)

\section{ACKNOWLEDGEMENT}

This paper was completed with the financial support by the Vietnam National Foundation for Science and Technology Development (NAFOSTED).

\section{REFERENCES}

[1] M. N. Semibratov, Technology of optical parts (in Russian), Moscow (1978).

[2] Nguyen Van Khang, Nguyen Trong Hung, Ninh Duc Ton, Improve processed surface's precision of optical elements by grinding under kinematic program control, Technische Mechanik, Band 28, Heft 2 (2008), 156 - 165.

[3] A. A. Shabana, John Wiley and Sons, Computational dynamics, New York (1989).

[4] E. J. Haug, Allyn and Bacon, Computer aided kinematics and dynamics of mechanical systems (1) Basic methods, Boston (1989).

[5] H. Josephs, R. L. Huston, Dynamics of mechanical systems, CRS Press, Boca Raton (2002).

[6] Schiehlen W., Technische Dynamik, Teubner, Stuttgart (1986).

[7] Nguyen Trong Hung, On the influences of some technological factors on the surface precision of optical parts in grinding (in Vietnamese), Ph.D. Thesis, Hanoi University of Technology (2003).

[8] Nguyen Van Khang, Nguyen Trong Hung, Nguyen Minh Phuong, The software for calculating kinematic program of optical parts, Department of Applied Mechanics, Hanoi University of Technology (2003).

Received May 17, 2010

\section{MộT THUẬT TOÁN MỚI VỀ ĐIỀU KHIỂN CHƯƠNG TRÌNH ĐộNG HỌC MÀI NGHIỀN CHI TIẾT QUANG}

Dựa trên các khái niệm hệ số phủ cục bộ, hệ số phủ trung bình, hệ số vận tốc khi mài nghiền các chi tiết quang và lý thuyết động học hệ nhiều vật, trong bài báo này đã trình bày một thuật toán điều khiển chương trình động học trong quá trình mài nghiền các chi tiết quang nhằm mục đích nâng cao độ chính xác và năng suất gia công. Sử dụng thuật toán này một phần mềm đã được xây dựng ở Trường Đại học Bách khoa Hà Nội. 